Editorial

Spinal Cord (2002) 40, 431. doi:10.1038/sj.sc.3101342

\title{
Sacral root stimulation
}

For more than 30 years electrical stimulation of peripheral nerves has been applied to restore or improve body functions such as walking, hearing, bladder voiding, and grasping. Electrical stimulation generates action potentials in stimulated nerves, which depending on the nerves' function, could cause contractions of the muscles, could elicit reflexes, or could help a certain group of patients to hear. Devices that apply electrical stimulation and are designed for Spinal Cord Injured (SCI) patients are used most frequently as orthoses/prostheses to assist these patients in performing various tasks in daily living activities, such as grasping and bladder voiding. The most successful electrical stimulation systems developed for SCI patients are devices that stimulate sacral roots. These systems are used to: (1) activate the detrusor muscle that causes bladder emptying; (2) activate urethral sphincter and pelvic floor muscles to reduce the number of incontinence episodes; or (3) stimulate various reflex pathways that participate in controlling lower urinary tract functions with the objective of suppressing or rectifying non-physiological behaviour of the lower urinary tract that occurs due to SCI.

Historically, the sacral anterior root stimulation system Vocare, developed by Brindley, and manufactured by Finetech Medical Ltd. (United Kingdom) and NeuroControl Co. (USA), represents the first truly successful electrical stimulation system. Over the last 20 years more than 2000 of these devices have been successfully implanted. The main feature of this stimulator is that it can produce bladder emptying on demand with minimal residual urine volume after bladder emptying. This, in turn, contributes to a significant reduction in the number of urinary tract infections SCI patients commonly experience. It should be mentioned that this system simultaneously contracts the detrusor muscle and the urethral sphincter by stimulating sacral roots. Curiously, even though both detrusor muscle and urethral sphincter are stimulated simultaneously (opposing functions), the bladder is successfully emptied. This is achieved due to the fact that the smooth muscle of the detrusor contracts and relaxes more slowly than the sphincter muscle. If the bursts of electric stimuli last only a few seconds and are applied intermittently, constant bladder pressure can be maintained during the stimulation intervals while the sphincter periodically relaxes allowing urine to flow in between stimulation bursts. Implantation of the Brindley system has been increasingly supplemented by surgical transection of the sacral sensory nerve roots to reduce hyper reflexia of the bladder (increasing bladder capacity and compliance), to reduce detrusor-sphincter dyssynergia (which improves urine flow), and to reduce reflex incontinence (which protects upper urinary tracts from uteric reflux and hydronephrosis). This intervention has certain drawbacks as well, such as abolition of reflex erection, reflex ejaculation, and reflex defecation. In recent years SCI patients have often objected to this intervention, not only because of the above drawbacks, but also because this intervention is irreversible. In the last 10 to 15 years researchers in the field of spinal cord repair have been promising that they will find the means of repairing the spinal cord after SCI in the forseeable future. In anticipation of this future medical treatment, SCI patients have been reluctant to endorse any treatment that might be irreversible.

Besides typical urinary tract problems that result from SCI, many disorders originate from excessive bladder contractions caused by the detrusor's 'instability', hyper-reflexia, and detrusor-sphincter dyssynergia. These disorders can cause complications such as persistent high intravesicular pressures, incontinence, and residual urine. A successful approach for resolving these problems is the stimulation of sacral afferent nerves that modulate reflexes and thus regulate perturbed urinary tract functions (neuromodulation). The implanted electric stimulator, Medtronic InterStim, manufactured by Medtronic Co. (USA), applies neuromodulation to sacral nerves via a wire electrode inserted in the sacral foramina and is able to correct successfully the inappropriate neural reflex behavior in more than $60 \%$ of patients implanted with the system.

Current research efforts are aimed at combining neuromodulation and bladder emptying using selective sacral posterior and anterior root stimulation. The objective is to develop a system that triggers neuromodulation and inhibits the bladder only when needed, i.e. when the onset of bladder contraction is observed. In this volume of Spinal Cord a number of original articles are presented which are discussing these and other new developments in the field of sacral root stimulation. 